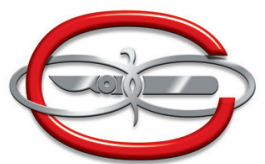

ASOCIACIÓN

COLOMBIANA

DE CIRUGÍA

\title{
Volver a empezar: cirugía electiva durante la pandemia del SARS-CoV2. Recomendaciones desde la Asociación Colombiana de Cirugía
}

\author{
Start over: Elective surgery during the SARS-CoV2 pandemic. \\ Recommendations from the Colombian Association of Surgery
}

Arnold José Barrios P. ${ }^{1}$, Robín Prieto², Lilian Torregrosa3 ${ }^{3}$, Carlos Álvarez4, Juan David Hernández ${ }^{5}$, Luis Gabriel González ${ }^{6}$, Álvaro Valencia ${ }^{7}$, Jorge Ospina ${ }^{8}$, Jorge Herrera ${ }^{9}$, John Henry Moore ${ }^{10}$, Juan Carlos Ayala11, Nayib Zurita ${ }^{12}$, Felipe Vargas ${ }^{13}$, Hernando Coba ${ }^{14}$, Roosevelt Fajardo ${ }^{15}$, Bernardo Borraez ${ }^{16}$, Manuel Moros ${ }^{17}$, Fredy Mendivelso ${ }^{18}$, Olga Aristizabal ${ }^{19}$, Fredy Guevara ${ }^{20}$, Felipe Cabrera ${ }^{21}$, Patrizio Petrone ${ }^{22}$

\footnotetext{
Médico, Especialista en Cirugía General. Jefe Departamento Quirúrgico de Clínica Colsanitas. Bogotá DC., Colombia

Médico, Especialista en Cirugía General, CEHYD SAS. Bogotá DC., Colombia

Médico, Especialista en Cirugía General, Cirugía de Mama y Tejidos Blandos, Magister en Bioética. Presidenta Asociación Colombiana de Cirugía. Directora Departamento de Cirugía y Especialidades, Facultad de Medicina, Pontificia Universidad Javeriana. Bogotá DC., Colombia

4 Médico, Especialista en Medicina Interna e Infectología. Clínica Colsanitas. Profesor titular Facultad de Medicina, Universidad Nacional de Colombia. Bogotá DC., Colombia.

5 Médico, Especialista en Cirugía General. Fundación Santa Fe de Bogotá. Bogotá, DC., Colombia

6 Médico, Especialista en Cirugía General. Hospital Central de la Policía Nacional. Hospital Universitario Nacional de Colombia. Bogotá DC., Colombia

Médico, Especialista en Cirugía General y Cirugía Bariátrica. Clínica Colsanitas. Bogotá DC., Colombia

8 Médico, Especialista en Cirugía General y Cirugía Vascular. Vicepresidente Médico, Clínica del Country. Bogotá DC., Colombia

9 Médico, Especialista en Cirugía General. Jefe de Cirugía, Clínica La Estancia. Popayán, Colombia

10 Médico, Especialista en Cirugía General. Director del Centro de Cirugía Ambulatoria CCA Bogotá DC., Colombia

11 Médico, Especialista en Cirugía General. Clínica del Country. Hospital Universitario Infantil de San José. Bogotá DC., Colombia

12 Médico, Especialista en Cirugía General. Profesor, Universidad del Sinú. Cartagena, Colombia.

13 Médico, Especialista en Cirugía General. Jefe de Clínicas Quirúrgicas Hospital Universitario Mayor Méderi. Bogotá DC., Colombia

14 Médico, Especialista en Cirugía General. Jefe, Programa de Cirugía General, Universidad de Cartagena. Cartagena, Colombia

15 Médico, Especialista en Cirugía General. Director de educación, Fundación Santa Fe de Bogotá. Bogotá, DC., Colombia

16 Médico, Especialista en Cirugía General y Cirugía Gastrointestinal y Endoscopia Digestiva. Departamento de Ciencias Clínicas, Universidad Tecnológica de Pereira. Pereira, Colombia

17 Médico, Especialista en Cirugía General. Clínica Gastroquirúrgica. Cúcuta, Colombia.

18 Médico, Especialista en Epidemiología. Magister en Epidemiología Clínica, Magister en Salud Pública, Magister en Bioestadística. FETP. Profesor, Facultad de Medicina, Fundación Universitaria Sanitas. Clínica Reina Sofía. Bogotá DC., Colombia

19 Médica, Especialista en Pediatría, Cuidado Intensivo y Epidemiología. Gerente General Clínica Reina Sofía. Bogotá DC., Colombia

20 Médico, Especialista en Medicina Interna e Infectología. Clínica Reina Sofía. Bogotá DC., Colombia

21 Médico, Especialista en Cirugía General. Universidad El Bosque. Bogotá DC., Colombia

22 Médico, Especialista en Cirugía General, Emergencias y Cuidado Intensivo. Profesor Asociado de Cirugía. NYU Long Island School of Medicine. NYU Winthrop Hospital. New York
}

Palabras clave: COVID-I9; virus SARS-CoV2; coronavirus; infecciones por coronavirus; pandemia; cirugía general; quirófanos.

Key words: COVID-I9; SARS-CoV2 virus; coronavirus; coronavirus infections; pandemics; general surgery; operating rooms.

Fecha de recibido: 06/05/2020 - Fecha de aceptación: 08/05/2020

Correspondencia: Arnold José Barrios. Jefe Departamento Quirúrgico de Clínica Colsanitas. Dirección: Calle 127 No. 20-56. Piso 3. Departamento de Cirugía, Teléfono: 3105748411. Correo electrónico: ajbarrios@colsanitas.com

Citar como: Barrios AJ, Prieto R, Torregrosa L, Álvarez C, Hernández JD, González LG, et al. Volver a empezar: cirugía electiva durante la pandemia del SARS-CoV2. Recomendaciones desde la Asociación Colombiana de Cirugía. Rev Colomb Cir. 2020;35:302-21. https://doi.org/10.30944/20117582.656

Este es un artículo de acceso abierto bajo una Licencia Creative Commons - BY-NC-ND https://creativecommons.org/licenses/by-ncnd/4.0/deed.es 


\section{Introducción}

La pandemia por el virus SARS-CoV-2 y la enfermedad generada (COVID-I9, del inglés Coronavirus 2019 Disease), llevó a nivel mundial a cambios significativos en la forma de prestar los servicios de salud, incluida la actividad quirúrgica. Como parte de esta realidad, se recomendó en Colombia la adopción de disposiciones enfocadas a preparar nuestro sistema de salud para enfrentar una avalancha de pacientes COVID-I9, que se esperaba fuera similar a la presentada en otros países $^{1,2}$. Las medidas se tomaron en el momento oportuno, ganando tiempo de preparación, sin que hasta la fecha (8 semanas después del primer caso diagnosticado en Colombia) se haya desbordado la demanda de atención, modificando la forma esperada de la curva epidemiológica, con un número menor de pacientes ${ }^{3}$.

Entre las medidas de preparación para la fase de mitigación, se contempla la expansión y adecuación de la infraestructura hospitalaria, priorizando las unidades de cuidado intensivo (UCI) y disminuyendo las hospitalizaciones por procedimientos quirúrgicos. Sin embargo, teniendo en cuenta que la dinámica de la epidemia se prolongará en el tiempo, se hace necesario establecer una estrategia que permita a las instituciones de salud llevar a cabo los procedimientos quirúrgicos, con prudencia y de manera segura, tanto para el paciente como para el equipo de salud, de acuerdo a la epidemiología del país y de cada región.

Para el reinicio seguro de las actividades quirúrgicas, es importante contar con un protocolo bien definido de evaluación de los pacientes, debido al aumento del riesgo de complicaciones posoperatorias observado en pacientes asintomáticos COVID-I9 positivos llevados a cirugía. La tamización clínica y la realización de pruebas rápidas o moleculares, cuya utilidad aún es controvertida, han buscado identificar ese grupo de pacientes asintomáticos; sin embargo, estas pruebas han llegado recientemente al país, no tienen disponibilidad general y su utilidad dependerá de la incidencia de casos durante la epidemia ${ }^{\mathrm{I}}$.

Las diferentes proyecciones muestran que el SARS-CoV2 llegó para acompañarnos por un tiempo largo aún no establecido; según modelos matemáticos, los brotes recurrentes pueden llegar hasta el año 2024 o $2025^{4}$. En el futuro, serán los entes estatales quienes se encarguen de suministrar los datos que confirmen la evolución del comportamiento en el ciclo epidemiológico y acorde a esto, establecer la reanudación progresiva de las actividades en salud.

Dado que a la fecha no hay certeza de un tratamiento efectivo o de la creación de una vacuna para la prevención del COVID-I9, los cambios que hemos adoptado en nuestra forma de ejercer la actividad quirúrgica serán parte de un aprendizaje, que quedará marcado como una impronta en nuestra nueva forma de vida.

Teniendo en cuenta las anteriores consideraciones y siendo necesario continuar con el manejo quirúrgico de algunas enfermedades, para evitar complicaciones, la Asociación Colombiana de Cirugía (ACC), a partir de la mejor información disponible en el momento a nivel global, genera las siguientes recomendaciones con el ánimo de orientar a los cirujanos e instituciones del país, para que empleando los recursos disponibles, puedan retomar las actividades quirúrgicas en los diferentes escenarios de atención de consulta externa y cirugía electiva. Este documento tiene como objetivo fundamental servir de apoyo para reanudar el funcionamiento de los servicios quirúrgicos de forma segura, protegiendo a nuestros pacientes mediante la reducción del riesgo de infección con rutas de circulación, quirófanos y áreas hospitalarias (incluidos consultorios y salas de espera) libres de COVID-I9. De igual manera, las recomendaciones se generan con la intención de salvaguardar a todo el personal asistencial, considerando que si éste se contagia, se convierte en una carga y a la vez, en una limitante para dar continuidad al funcionamiento eficiente de los servicios de salud.

\section{Consideraciones epidemiológicas y definiciones}

Ante la reapertura económica con el esquema de acordeón propuesto por el gobierno, se espera un comportamiento oscilante de la pandemia. Las directrices frente a cierre y apertura de servicios 
de cirugía electiva no prioritaria pueden cambiar, en razón de modificaciones en la situación local de la enfermedad y la saturación del sistema hospitalario. Cada institución deberá estar atenta a estos cambios y tomar las decisiones oportunas que se requieran. Para este documento planteamos las siguientes definiciones:

- Cirugía electiva: es aquella en la que se dispone del tiempo necesario para evaluar cuidadosamente los riesgos y beneficios del procedimiento, permitiendo una programación planificada.

- Morbilidad: es la frecuencia de la enfermedad en proporción a una población.

- Positividad: determina cuál es el porcentaje de muestras positivas con respecto al total de las muestras procesadas.

- Ocupación de camas en UCI: mide la proporción de camas de cuidados intensivos ocupadas con respecto al total de camas existentes, independientemente de si son ocupadas por pacientes con o sin COVID-I9.

- Ocupación hospitalaria: es el número de camas hospitalarias ocupadas sobre camas instaladas en un periodo determinado, expresado en porcentaje o razón.

\section{Principios para reabrir cirugía electiva}

Antes de dar inicio a las actividades de cirugía electiva, se debe verificar que se cuenta con todos los requisitos para asegurar una mitigación de los riesgos para el personal de salud y los pacientes involucrados en estas atenciones. Se recomienda conformar un equipo institucional que lidere este proceso, evalúe el cumplimiento de los requisitos y posteriormente haga un seguimiento estricto de la evolución de las medidas asumidas a nivel institucional y local 5 .

La institución debe tener disponibilidad de recursos, los que deben incluir suficiencia de camas hospitalarias y de UCI, respiradores, recurso humano capacitado y elementos de protección personal (EPP) adecuados para la atención de todos los pacientes quirúrgicos ${ }^{6}$. Los requisitos que se deben tener en cuenta respecto a la preparación institucional incluyen:

- Tener rutas claras y protocolos de manejo perioperatorio específicos para pacientes sospechosos o confirmados COVID-I9 y pacientes sin COVID-I9, incluidos los protocolos para el manejo de la sala quirúrgica asignada a casos sospechosos o positivos.

- Tener la infraestructura hospitalaria en salas de cirugía, recuperación y UCI que permita la atención de estos pacientes en áreas diferenciales.

- Tener áreas de Hospitalización claramente diferenciadas para paciente COVID-I9 y No COVID-I9 con protocolos de manejo específicos para cada una.

Teniendo en cuenta el recurso hospitalario, específicamente la cantidad de camas destinadas para las dos poblaciones arriba enunciadas, se sugieren las siguientes fases que pueden orientar para definir la programación de la cirugía electiva:

- Fase I o de alerta mínima: la programación de cirugía será normal.

- Fase II o de alerta leve: la programación de cirugía dependerá de la necesidad y disponibilidad de UCI posoperatoria para realizar el procedimiento.

- Fase III o de alerta moderada: solo se programarán procedimientos electivos que no requieran UCI o que su riesgo sea mínimo.

- Fase IV o de alerta alta: no se realizarán procedimientos electivos. Solamente se realizarán procedimientos de Urgencia o Emergencia.

La tabla I muestra los escenarios o fases en los cuales se puede desarrollar la pandemia al interior de las instituciones y las recomendaciones frente a las actividades quirúrgicas según la ocupación de camas por pacientes COVID-I9. 
Tabla 1. Escenarios institucionales para el manejo de cirugía programada

\begin{tabular}{|c|c|c|c|c|}
\hline \multirow{3}{*}{ Escenarios } & \multicolumn{4}{|c|}{ Fase } \\
\hline & I & II & III & IV \\
\hline & Alerta mínima & Alerta leve & Alerta media & Alerta alta \\
\hline $\begin{array}{l}\text { Porcentaje }(\%) \text { de ocupación de la } \\
\text { UCl con pacientes COVID-19 [+] }\end{array}$ & $0-15 \%$ & $15-30 \%$ & $30-70 \%$ & $\geq 70 \%$ \\
\hline $\begin{array}{l}\text { Porcentaje }(\%) \text { de ocupación de } \\
\text { hospitalización con pacientes } \\
\text { COVID-19 [+] }\end{array}$ & $<10 \%$ & $10-30 \%$ & $30-60 \%$ & $\geq 60 \%$ \\
\hline Actividad quirúrgica & Normal & $\begin{array}{l}\text { Pacientes que po- } \\
\text { drían requerir } \mathrm{UCI}\end{array}$ & $\begin{array}{l}\text { Cirugía de mínimo } \\
\text { riesgo de UCI, te- } \\
\text { niendo pisos exclu- } \\
\text { sivos No COVID-19 } \\
\text { disponibles }\end{array}$ & $\begin{array}{l}\text { Solo cirugía prio- } \\
\text { ritaria y cirugía de } \\
\text { urgencia no dife- } \\
\text { rible }\end{array}$ \\
\hline
\end{tabular}

Fuente: Elaboración de los autores UCI: Unidad de Cuidados Intensivos

Se debe contar con un protocolo institucional para la priorización de pacientes por parte del grupo quirúrgico, que permita definir a nivel individual, la pertinencia de intervenir a cada paciente. Se recomienda el uso de la escala de estratificación MeNTS (Medically Necessary Time Sensitive), propuesta por el American College of Surgeons (ACS) ${ }^{7}$, que fue creada específicamente para apoyar la toma de decisiones quirúrgicas en el escenario de la pandemia de COVID-I9. Contempla tanto la optimización del manejo de recursos hospitalarios en épocas de escasez, como los riesgos particulares de cada paciente, la prioridad de la patología que origina la indicación quirúrgica y la complejidad del procedimiento quirúrgico.

Al aplicar la escala MeNTS, una puntuación baja plantea que la cirugía es definitivamente recomendable y por el contrario, una puntuación elevada quiere decir que llevar a cabo dicha intervención durante la pandemia, no es la estrategia más eficiente y adecuada y se debe reconsiderar el momento de llevarla a cabo. Como punto de corte, el ACS propone un valor de 55-57 para reconsiderar la realización del procedimiento quirúrgico en el paciente evaluado y la Asociación Española de Cirujanos recomienda un valor de 6o puntos. Por supuesto la aplicación de la escala no reemplaza el juicio clínico del cirujano, más bien supone una valoración individualizada y meticulosa de cada paciente, que permita la toma de decisiones conjunta con el enfermo, quien debe tener muy claros los riesgos particulares de ser operado durante la pandemia y así debe quedar registrado en la historia clínica. En casos particulares será útil citar una junta de varios especialistas para la discusión del caso y en situaciones de mayor complejidad se debe acudir a la instancia de un Comité de ética.

\section{Principios}

Las guías de cada región deben ajustarse al comportamiento local de la enfermedad, para lo cual se tendrán en cuenta los siguientes principios:

- Consenso y respaldo de las asociaciones científicas para una apertura ordenada de actividades, acorde al riesgo de cada procedimiento.

- Disponibilidad real y suficiente de recursos hospitalarios, incluyendo camas de UCI, camas hospitalarias y EPP.

- Recurso humano suficiente, capacitado, con evaluación y soporte psicológico, definido por protocolo institucional.

- Tener disponibilidad de pruebas diagnósticas suficientes para pacientes y personal de salud, cuando se considere necesario su uso. 


\section{Tamización y pruebas diagnósticas}

La incertidumbre que genera la variabilidad de esta enfermedad, lleva a plantear retos que enfrentan al paciente y a su grupo tratante con la realidad de la enfermedad y la muerte. Es necesario generar estrategias que disminuyan este temor y permitan tomar las conductas más seguras, especialmente ante los datos aun no claros de la sensibilidad y especificidad de los métodos diagnósticos. Se debe mantener un proceso de evaluación escalonado, que permita clasificar a cada paciente, para seguir un mapa de decisiones y determinar el momento adecuado de la cirugía $^{6,8,9}$.

Tamización clínica. Busca signos y síntomas de la enfermedad, que deben explorarse como una lista de chequeo, evaluando la presencia de:

- Fiebre

- Odinofagia

- Anosmia

- Disgeusia

- Tos seca

- Disnea

- Adinamia o fatiga

- Síntomas gastrointestinales (por ejemplo, nausea o diarrea)

- Antecedente de confinamiento

- Contacto en los últimos I4 días con paciente confirmado / sospechoso o persona con síntomas

Al hacer la evaluación clínica de los pacientes en la consulta prequirúrgica, preanestésica o en la evaluación de tamización para la preparación de la cirugía, la presencia de cualquiera de los síntomas o contactos anteriormente mencionados, hacen que el paciente no sea candidato para cirugía electiva en ese momento. Todos estos pacientes requerirán un aisla- miento social (físico) por un mínimo de I4 días. Durante el interrogatorio debe buscarse antecedentes de inmunosupresión, VIH, cáncer y manejo con quimioterapia, diabetes, hipertensión, obesidad, falla renal, hipotiroidismo, malnutrición, uso de corticoides, enfermedad pulmonar obstructiva crónica (EPOC), asma o tabaquismo; ya que este grupo de pacientes tienen mayor riesgo de morbimortalidad y de requerir UCI en caso de estar infectados con SARS-CoV-2.

Para evaluar a los pacientes asintomáticos, debe correlacionarse la tamización clínica con las pruebas de laboratorio, con el objetivo de proteger al potencial paciente y al equipo quirúrgico. Las pruebas han mostrado variabilidad en su calidad, generando controversias sobre cuál, cómo y cuándo usarlas y cómo interpretarlas.

Estas pruebas están recomendadas cuando la prevalencia de la enfermedad sea alta (consideramos que mayor del I5 \%), por lo que la extrapolación de datos europeos o norteamericanos en este momento de la pandemia en Colombia, puede ser una estrategia no acertada, por la diferencia en la incidencia actual entre los países. Las pruebas para tamización de SARS-CoV2 se pueden dividir en dos grupos:

A. Moleculares (RT-PCR): detectan el ARN del virus por medio de amplificación en reacción de cadena de polimerasa (PCR). Las muestras deben ser obtenidas mediante hisopado nasofaríngeo u orofaríngeo. Se consideran estas pruebas como "la prueba de oro" en casos agudos.

B. Serológicas: indican la respuesta del huésped al virus; entre ellas se encuentran los anticuerpos de tipo IgM e IgG. Para la programación quirúrgica, la pregunta trascendental es si el paciente programado y asintomático puede ser portador de la enfermedad, por lo cual es pertinente tamizar pacientes en búsqueda de los asintomáticos, de ahí la relevancia de solicitar anticuerpos que orienten la toma de decisiones bajo una secuencia lógica. 
Una vez realizada la evaluación previa a la cirugía, se observa que ninguna información, individual o en conjunto, ofrece la certeza deseada sobre la relación específica de un paciente con la infección por SARS-CoV-2. En estos escenarios el interrogatorio, la credibilidad del paciente, la búsqueda de los síntomas relacionados, el momento en que inician dichos síntomas, la epidemiología local y la interpretación de las pruebas rápidas (cuando estén disponibles), nos brindarán mayor seguridad, sin que esto signifique descuidar el adecuado seguimiento de los protocolos y el uso de los EPP ${ }^{6}$.

Si la tamización es positiva (presencia de alguno de los signos o síntomas) se debe cancelar la programación del procedimiento, realizar la evaluación correspondiente con estudios de PCR y de acuerdo con el resultado de las pruebas y la resolución de los síntomas, revaluar al paciente una vez se encuentre completamente recuperado, para proceder a programar su cirugía.

Ante la carencia de pruebas rápidas en una institución y en el contexto de un paciente completamente asintomático, que haya cumplido con el confinamiento y no tenga antecedente de contacto con un paciente COVID-I9 sospechoso o confirmado sin la protección adecuada, es razonable continuar con la programación de la cirugía, sin que constituya un requisito la realización de pruebas. Si en la institución hay acceso a las pruebas rápidas IgG e IgM ${ }^{10}$ y consenso sobre su uso en este escenario, se recomienda realizarlas 24 horas antes de la cirugía, o de acuerdo al protocolo particular de la institución, con la siguiente interpretación:

- Si el paciente tiene una IgM [-] con IgG [+] (paciente recuperado) o IgM [-] con IgG [-] (paciente sano sin inmunidad): se llevará a cirugía.

- Si el paciente tiene una IgM [+] con IgG [+] o IgM [+] con IgG [-] (paciente con infección activa): se debe cancelar el procedimiento y se definirá la realización de una PCR (figura I).

Se recomienda, que en caso de que la prevalencia de la enfermedad supere el $15 \%$ de la población, se instaure la evaluación con pruebas en todos los pacientes.

El personal de salud debe ser sometido a tamización clínica y las estrategias adicionales que cada institución contemple (lo cual puede incluir evaluaciones clínicas y/o pruebas de anticuerpos periódicas). Se debe incentivar el reporte de signos y síntomas, así como de la ocurrencia de contactos estrechos no protegidos con pacientes COVID-I9 confirmados o sospechosos. En estos casos se deberán realizar pruebas serológicas y ante un resultado negativo, se deberá valorar la repetición en función de la evolución de los escenarios ${ }^{6, \mathrm{IO}-12}$.

\section{Recomendaciones para uso de EPP y acciones de protección personal (APP)}

La situación actual ha hecho que el uso de EPP sea visto de una manera diferente. Es claro que a consecuencia del SARS-CoV-2, la protección del personal de la salud y de los pacientes deberá ser mayor a la que venía empleándose antes de la pandemia y se mantendrá por un periodo de tiempo aún no establecido. Entender los mecanismos de transmisión del virus es muy importante, tanto para el manejo de los pacientes como para el adecuado uso de los EPP ${ }^{6}$. La transmisión del virus se produce por la entrada de este a las vías respiratorias, mucosas y conjuntivas, a través de gotas o aerosoles; no se trasmite por contacto directo a través de la piel o la sangre. Genera inquietud la detección del virus en líquido peritoneal y heces, sin conocer si estos virus puedan tener potencial infeccioso ${ }^{13}$.

Es trascendental evaluar los momentos de mayor potencial de trasmisión, que pueden ser divididos en pre, intra y posoperatorio. $\mathrm{Al}$ ingreso a la institución en el preoperatorio, el contagio del personal de salud se ha presentado con más frecuencia fuera de los lugares de atención a los pacientes, por ejemplo cafeterías y sitios donde se produce contacto comunitario. Teniendo en cuenta las distintas áreas en las que deberán interactuar pacientes y personal de salud, en la tabla 2 se presentan los EPP y las acciones necesarias para la protección personal. 


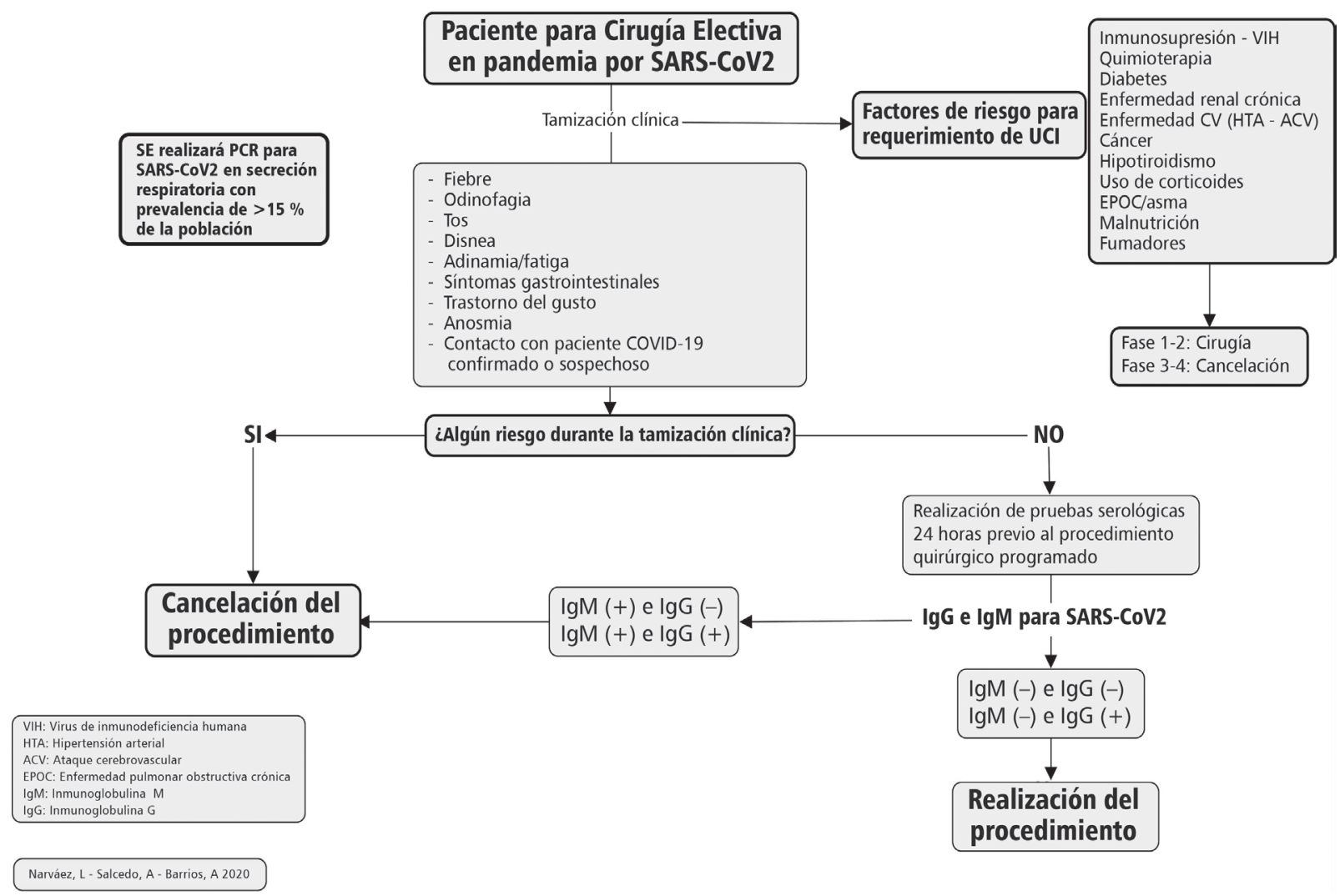

Figura 1. Flujograma para tamización clínica y pruebas de laboratorio a pacientes prequirúrgicos Fuente: Elaboración de los autores.

Tabla 2. Recomendaciones para uso de equipos de protección personal (EPP) y acciones de protección personal (APP)

\begin{tabular}{lccccc}
\hline \multicolumn{1}{c}{ EPP y APP } & $\begin{array}{c}\text { Consulta } \\
\text { externa }\end{array}$ & $\begin{array}{c}\text { Ingreso a } \\
\text { cirugía }\end{array}$ & $\begin{array}{c}\text { Vestier de } \\
\text { pacientes y personal }\end{array}$ & $\begin{array}{c}\text { Salas de } \\
\text { cirugía }\end{array}$ & $\begin{array}{c}\text { Recuperación } \\
\text { y egreso }\end{array}$ \\
\hline Lavado de manos & PS - Pac & PS - Pac & PS - Pac & PS & PS \\
Mascarilla quirúrgica & PS - Pac & PS - Pac & PS - Pac & Pac & Pac \\
Mascarilla N95 & - & - & - & EQ - An & PS \\
Visor & PS & - & - & An - PS & PS \\
Monogafas & - & - & - & EQ - An & PS \\
Guantes & PS (examen) & - & - & EQ - An - PS & PS \\
Bata anti fluido & - & - & - & EQ - An & PS \\
Gorro & - & - & - & EQ - An - PS & PS \\
Calzado/polainas & - & - & - & EQ - An - PS & PS \\
\hline
\end{tabular}

PS: personal de salud, incluye administrativos, servicios generales

Pac: paciente y acompañante

EQ: equipo quirúrgico (auxiliar, instrumentadora, cirujano, ayudante)

An: equipo de anestesia (anestesiólogo, ayudante, enfermera) 
Los EPP se dividen en dos, que se complementan ${ }^{6}$. Por un lado está la protección para gotas y aerosoles empleando mascarillas, monogafas y/o visores. Por el otro lado, está la protección del riesgo por contacto, para lo cual se emplea el lavado de manos (que debe ser realizado de 40 a 60 segundos, de manera frecuente) o la desinfección de manos con alcohol glicerinado (como alternativa, sin que reemplace el lavado de manos). La institución debe garantizar la disponibilidad de instalaciones para este fin. Los desinfectantes para las manos deben estar disponibles en el lugar de atención ambulatoria o de examen, así como en los quirófanos. Para las superficies y equipos, la limpieza con amonio cuaternario debe ser la práctica, sumada al uso de guantes, bata, gorro y calzado cerrado exclusivo, protegido con polainas.

Deben implementarse protocolos para la colocación y retiro de los EPP, ya que son momentos de alta movilidad de partículas y potencial transmisión, en lo posible supervisados por una persona espejo entrenada previamente. La promoción de un clima de seguridad institucional ayuda a mejorar la adhesión a las medidas recomendadas y por lo tanto a la reducción de los riesgos posteriores. La provisión de personal y suministros adecuados, junto con liderazgo y educación del personal sanitario, los pacientes y las visitas, son fundamentales para un mejor clima de seguridad en los entornos de la atención de salud ${ }^{14-16}$.

En el posoperatorio el mayor riesgo de contaminación se presenta durante el retiro de los EPP, usualmente cuando el equipo quirúrgico ha disminuido la atención por haber finalizado el procedimiento. Por lo tanto, mientras más elementos utilice, hay más dificultades técnicas y más riesgo de contagio. El uso de elementos de protección debe ser racional, medido de acuerdo con el riesgo y los recursos disponibles (figura 2).

\section{Recomendaciones institucionales locativas}

Dentro del hospital se deben tener las siguientes consideraciones ${ }^{17-19}$ :

- El hospital debe contar con protocolos que minimicen el riesgo de transmisión de la enfermedad en áreas de ingreso, quirófanos, recuperación y egreso de los pacientes.

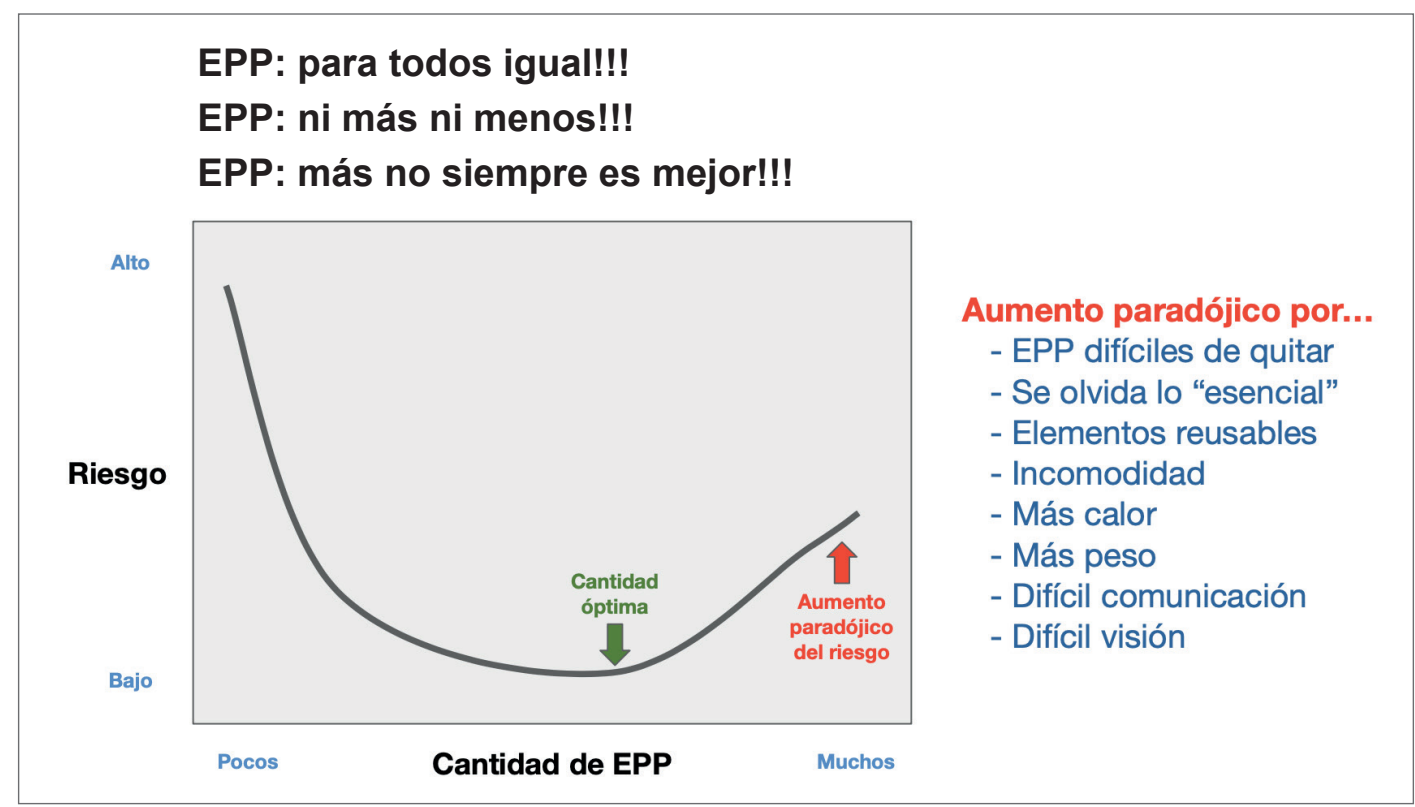

Figura 2. Uso suficiente y adecuado de equipos de protección personal (EPP) 
- El hospital debe garantizar rutas de circulación, de trasporte de equipos e instrumental y de manejo de desechos que no generen riesgo.

- El ingreso y atención de los pacientes a cirugía programada, debe ser diferenciada de la cirugía de pacientes COVID-I9 sospechosos o confirmados, para minimizar el riesgo de infección.

- Requisito de aislamiento físico en áreas destinadas para paciente COVID-I9 positivos.

- Las instituciones que cuenten con unidades de cirugía ambulatoria no deben recibir pacientes COVID-I9 positivos y deben aplicar protocolos definidos para tamizar los pacientes en búsqueda de los casos asintomáticos.

- Determinar según el tipo de salas, el recambio del aire que permita definir el tiempo entre procedimientos y los momentos de circulación en el quirófano.

- Asegurar la disponibilidad de instalaciones suficientes para el lavado de las manos.

- Tener disponibilidad en el lugar de atención de productos para higiene de las manos (agua limpia, jabón, toallas desechables, gel desinfectante).

- Inhabilitar las salas de espera, de esta manera, la visita al paciente se concentrará en la habitación del mismo y no en áreas comunes.

\section{Recomendaciones del comité multidisciplinario de quirófanos}

La seguridad del quirófano se basa en el equilibrio entre la complejidad de atención quirúrgica y la eficiencia. Los riesgos biológicos siempre estarán presentes, con o sin pandemia, y las salas de cirugía deben estar preparadas para funcionar en momentos de crisis. Esta eficiencia debe medirse, entre otras, por la productividad económica, pero durante la crisis, el indicador más apropiado será la presencia de personal contagiado.
El comité de quirófanos debe constituirse como un órgano multidisciplinario que tiene como objeto velar por el funcionamiento de un área compleja. Durante la pandemia es indispensable poner en funcionamiento cambios en la operatividad del área, por lo tanto, su funcionamiento debe tener una gobernanza colegiada ${ }^{7,20-24}$. Las funciones de este comité deben incluir:

- Modificar la distribución de las jornadas quirúrgicas, con el objeto de establecer la programación de los casos que deben realizarse, teniendo en cuenta la prioridad, la complejidad quirúrgica y el estado del paciente, dependiendo de la fase de la pandemia o el escenario en que se encuentre la institución.

- Definir los horarios, teniendo en cuenta la recomendación de prolongar los tiempos quirúrgicos, por lo cual se puede considerar la extensión del tiempo de funcionamiento durante las horas de la noche y los fines de semana, para responder a la demanda de cirugía electiva.

- Realizar una programación escalonada para evitar aglomeraciones.

- Tener en cuenta la presencia de casos de emergencia y prever la capacidad de las instalaciones para la atención de estas.

- Verificar que se hace tamización al personal del área quirúrgica, de acuerdo con las pruebas definidas por la institución, así como el análisis de sus resultados.

- Establecer protocolos de verificación de equipos y cambio de filtros de los sistemas de ventilación.

- Definir qué elementos personales pueden ingresar a salas de cirugía, recomendando abstenerse de permitir la entrada de celulares, equipos de sonido o cómputo; restringiendo la entrada solo a los elementos indispensables para la práctica del procedimiento. 
- Recomendar la disponibilidad de personal de apoyo para circular los diferentes quirófanos, desestimando la entrada y salida continua de la circulante de la sala, disminuyendo así la turbulencia del quirófano y la posibilidad de contaminación cruzada.

- Definir los protocolos quirúrgicos y anestésicos, antes de iniciarse el procedimiento, para disminuir la rotación de elementos en la sala.

- Definir con el servicio de patología las normas de embalaje y trasporte de las muestras. Durante la pandemia suprimir el uso de biopsias por congelación.

- Vigilar y exigir el cumplimiento de las listas de seguridad para el paciente.

- Supervisar los consentimientos informados debidamente diligenciados.

- Preservar la disponibilidad de unidades de apoyo, como UCI, laboratorio e imágenes diagnósticas, evitando mezclar los pacientes programados con los pacientes COVID-I9.

- Las instituciones deben tener e implementar una política de distanciamiento social para el personal, pacientes y visitantes de pacientes en áreas no restringidas, que cumpla con las recomendaciones locales y nacionales vigentes en ese momento para las prácticas de aislamiento comunitario.

- Velar por el adecuado estado de los equipos que minimicen la exposición al gas o el humo (neumoinsufladores con filtros) o pantallas que mejoren la visión en presencia de humo.

Junto con el comité de infecciones:

- Coordinar las políticas de limpieza de las instalaciones en el contexto de COVID-I9, que deben abordarse en la totalidad de las áreas durante el tiempo de atención.

- Elaborar el plan de emergencias del área.

- Establecer y supervisar los protocolos de limpieza y de bioseguridad.
- Definir el uso de ducha hospitalaria en los casos que sea necesario.

- Restringir el uso del vestido de calle y de calzado dentro de la institución hospitalaria, donde debe ser utilizado el vestido y calzado de dotación. Recomendamos seguir la instrucción de cuidado del personal de salud al llegar a sus casas.

- Los gorros quirúrgicos deben ser proveídos por la institución, evitando el uso de gorros extra institucionales.

- Minimizar el uso de dispositivos que generen humo y aerosoles.

- Restringir la circulación del personal extra institucional

\section{Recomendaciones en las diferentes fases del proceso de atención}

\section{Fase preoperatoria:}

- Evaluar las listas de pacientes cancelados y priorizar su atención, según su patología quirúrgica de base.

- Reprogramar siguiendo las recomendaciones previamente descritas.

- Ante listas de espera prolongadas, definir la necesidad de reconsulta, presencial o virtual, para evaluar la indicación de nuevos exámenes o exámenes complementarios.

- Organizar bloques quirúrgicos que puedan realizarse con anestesia local o regional, disminuyendo la generación de aerosoles durante la anestesia.

- Diseñar estrategias de educación virtual para el paciente, encaminadas a estimular el autocuidado y el conocimiento de su patología.

- Disminuir el tiempo de espera del paciente dentro de la institución previo a su cirugía, de acuerdo con los protocolos institucionales ${ }^{10,25-33}$. 
- Diligenciar y firmar el anexo del consentimiento donde se informan los riesgos relacionados con la pandemia (anexo I).

\section{Fase operatoria:}

- Mientras se determina el control de la pandemia en el país, todo el personal de la sala de cirugía tendrá que usar EPP completo.

- Respecto al abordaje quirúrgico (cirugía abierta o cirugía laparoscópica), el cirujano elegirá la vía de acuerdo con su pericia. No existe evidencia que permita recomendar el no hacer cirugía laparoscópica. Debe ser seguida la información en relación con los dispositivos de energía avanzada, la cual a la fecha no es concluyente.

- En cirugía laparoscópica se recomienda el uso de un circuito cerrado de eliminación del gas y del humo, para disminuir el riesgo de exposición.

- Emplear presiones bajas de neumoperitoneo, para lo cual es indispensable una adecuada relajación del paciente.

- Preferir trocares desechables con sistema de seguridad o de balón que brindan un sello más hermético.

- Realizar incisiones pequeñas ajustadas al trocar.

- Manipular cuidadosamente los instrumentos.

- El cirujano determinará, según el manual de delegación de funciones, el momento y caso que pueda asumir el residente en entrenamiento, bajo las mismas recomendaciones de cuidado de todo el equipo quirúrgico.

- Extraer trocares y especímenes quirúrgicos después de evacuar por completo el neumoperitoneo.

- Se recomienda el cierre de las heridas quirúrgicas con material que no requiera ser retirado (como cianoacrilato o suturas monofilamento absorbibles a corto plazo), así como evitar el uso de drenes o mechas, lo cual facilitará el control no presencial del paciente.

\section{Fase posoperatoria:}

- Se recomienda que el control posquirúrgico se realice de manera virtual cuando sea posible, en los tiempos que determinen los protocolos institucionales. Se generarán instructivos para los pacientes, quienes deben mantener un aislamiento preventivo por 2 semanas.

- El seguimiento telefónico se puede poner en marcha desde el preoperatorio, lo cual permite contacto directo con el paciente y su familia.

\section{Unidades de cirugía ambulatoria mayor}

La cirugía ambulatoria mayor (CAM) es una estrategia que debe ser considerada, ya que puede contribuir a disminuir los riesgos de las complicaciones (infecciosas y no infecciosas) generadas por la estancia hospitalaria y a favorecer la disponibilidad de camas a nivel local y regional durante la pandemia. Las "zonas No COVID-I9" pueden tener un impacto favorable frente al riesgo de contagio de los pacientes que se atienden $y$ el personal de salud involucrado en estas atenciones ${ }^{17,34}$. Por este motivo se recomienda:

- Considerar instituciones o servicios de cirugía ambulatoria mayor (CAM) debidamente habilitados, como "zonas No COVID-I9", para la atención de procedimientos de mediana complejidad, cumpliendo todas las normas relacionadas con la disponibilidad y el uso de EPP en estos escenarios ${ }^{6}$.

- Asegurar en estas instituciones o servicios, un plan de atención quirúrgica para la reanudación de las actividades, donde se garantice un adecuado proceso de evaluación y de selección de pacientes, en el que se incluyan como mínimo cuestionarios para la evaluación de signos y síntomas relacionados a la infección por COVID-I9. 
- Evaluar permanentemente los indicadores de calidad institucional.

- Garantizar un adecuado seguimiento posoperatorio, incluido un programa de atención domiciliaria en los casos que se requiera.

\section{Atención domiciliaria}

El uso de programas de atención domiciliaria está documentado en nuestro sistema de salud y permite llevar rápidamente a su casa a los pacientes que lo requieran, para continuar de esta forma la supervisión profesional. Esto favorece liberar camas hospitalarias y disminuir el riesgo de contagio. Por lo anterior, esta práctica debe ser estimulada, siguiendo los parámetros de bioseguridad aprobados para este escenario en el país ${ }^{6}$.

\section{Recomendaciones médico-legales}

Es muy importante que las acciones durante esta pandemia se encuentren avaladas por una asociación científica que proponga pautas, para que dentro de la autonomía de los profesionales sean adoptadas con fines de protección ante eventuales demandas. En este caso puntual debe aclararse que no existe al momento información con evidencia suficiente, para determinar de manera clara cuáles deberían ser las conductas ideales en cada caso. Este documento recomienda:

- Mantener una adecuada relación entre el médico y el paciente, así como una fluida línea de comunicación.

- Apoyar decisiones en juntas médicas multidisciplinarias.

- Involucrar a los pacientes y familiares en las decisiones médico-quirúrgicas.

- Incluir a los comités de ética médica en la toma de decisiones complejas, cuando esto aplique.

- Anexar un consentimiento informado específico con respecto al contagio y desenlaces inesperados generados por la enfermedad
COVID-I9, en todos los actos médicos realizados (anexo I)

- Establecer lineamientos para la prevención y reporte de accidentes por exposición ocupacional.

- Adherirse a las recomendaciones de los entes de control estatal para la limitación de algunos tipos de cirugía.

\section{Vigilancia del recurso humano}

Durante la progresiva reducción o escalamiento de las fases de la pandemia o escenarios, se deberán mantener los mejores procesos de vigilancia, para garantizar la seguridad de los profesionales. La pandemia exige que todas las estrategias busquen proteger al profesional, limitando la infección cruzada y preservando los procesos de atención. Las reuniones académicas, juntas del servicio y la constante actualización de la información, debe ser realizada de forma virtual para evitar los encuentros presenciales y el riesgo que esto implica.

Es importante conocer las comorbilidades y la edad de los miembros del equipo quirúrgico, con el fin de estratificar sus riesgos y establecer las medidas de protección apropiadas en forma individual. Los profesionales de la salud que se clasifiquen como caso COVID-I9 posible o confirmado, deberán tener un protocolo especial de seguimiento y manejo de acuerdo con el documento técnico del Ministerio de Salud ${ }^{35}$.

La institución debe definir una rotación adecuada del personal para evitar la sobre-exposición.

\section{Evaluación de niveles de estrés y fatiga en trabajadores sanos}

En la actual situación de pandemia, el personal de salud puede presentar frecuentemente estados de preocupación o de ansiedad, que alteran su estabilidad mental, por lo cual se recomienda ${ }^{36}$ :

- Establecer seguimiento para el personal de salud, definiendo estrategias de detección de conductas emocionales atípicas, que 
permitan hallar alteraciones del estado emocional.

- Implementar políticas de salud mental para el grupo asistencial, afianzando liderazgos, coordinando planes de acción entre la institución y la ARL, definiendo seguimientos, estableciendo y socializando la ruta de salud mental.

- Promover reconocimientos y agradecimientos al personal de salud, en forma permanente, por diferentes canales de comunicación.

- Flexibilizar los horarios para personal con afectación emocional o con familiares contagiados.

\section{Escenarios de atención e información a pacientes}

Telemedicina: cada institución deberá garantizar y reglamentar la prestación de este servicio y su debido ajuste a las normas gubernamentales y de ética médica.

Consulta externa presencial: esta se prestará a pacientes cuya presencia o examen clínico sea indispensables para el acto médico. En este escenario los prestadores de salud podrán atender a los usuarios siguiendo las recomendaciones de distanciamiento social preventivo, como son: citas espaciadas, salas de espera amplias, ubicación de pacientes a dos metros de distancia, uso de EPP para pacientes y personal médico asistencial, además de mantener las recomendaciones institucionales para la prevención de contagio.

Procedimientos diagnósticos y terapéuticos: la necesidad de un procedimiento diagnóstico y/o terapéutico requiere de una decisión concertada previamente con el paciente, donde se establezca la prioridad, sus riesgos y beneficios, y las consecuencias que esto implica.

Ingresos hospitalarios: el ingreso hospitalario se debe restringir a pacientes que estrictamente lo requieran. Las visitas de familiares de edad avanzada, embarazadas, o con comorbilidades de riesgo, deben ser abolidas.
Restringir el número y horarios de visita: cada institución debe definir el número de visitantes, el tiempo y los horarios de la visita.

\section{Consideracion final}

La dinámica de la pandemia, el conocimiento de la enfermedad, y la prestación de los servicios son variables, lo que obliga a una permanente actualización de la información.

\section{Elaborado y avalado por:}

Grupo de trabajo "Cirugía-ACC-Covid-ı9" de la Asociación Colombiana de Cirugía.

Todos los autores colaboraron en el diseño, publicación y divulgación de estos resúmenes visuales (visual abstract), los cuales consideramos de gran utilidad para la comunidad médica durante la actual pandemia COVID-I9 (anexo 2).

\section{Cumplimiento de normas éticas}

Consentimiento informado: Esta publicación es una revisión de la literatura, y como tal no hay necesidad de un consentimiento informado ni de aprobación del Comité de Ética Institucional.

Conflicto de intereses: Ninguno declarado por los autores.

\section{Referencias}

I. Cuevas-López L, Acosta JCA, Velásquez-Jiménez OA, Navarro-Alean4 JA, González-Higuera LG, Medrano NZ, et al. Recomendaciones para el manejo de los pacientes quirúrgicos urgentes durante la pandemia COVID-I9. Rev Colomb Cirugía. 2020;35:I43-I52. https://doi.org/IO.30944/201I7582.6I9

2. Balibrea JM, Badia JM, Rubio Perez I, Martin Antona E, Alvarez Pena E, Garcia Botella S, et al. Manejo quirúrgico de pacientes con infección por COVID-I9. Recomendaciones de la Asociación Española de Cirujanos. Cir Esp. 2020 Apr;I-9. https://doi.org/IO.IOI6/j.ciresp.2020.03.00I

3. Jianxi L. When will COVID-I9 end? Answer from data-driven innovation lab. Singapore University of Technology and Design. IFIA International Federation of Inventors' Associations. 2020. Disponible en: https://www.ifia.com/news/when-will-covid-I9-end/

4. Kissler SM, Tedijanto C, Goldstein E, Grad YH, Lipsitch M. Projecting the transmission dynamics of SARS$\mathrm{CoV}-2$ through the postpandemic period. Science. 2020 Apr;I4. https://doi.org/IO.II26/science.abb5793 
5. Asociación Colombiana de Infectología (ACIN) e Instituto de Evaluación de Nuevas Tecnologías de la Salud (IETS). Consenso colombiano de atención, diagnóstico y manejo de la infección por SARS-COV-2/COVID-I9 en establecimientos de atención de la salud. Recomendaciones basadas en consenso de expertos e informadas en la evidencia. Infectio. 2020;24(3)Suplemento. https://doi.org/IO.22354/in.v24i3.85I

6. Rodríguez M, Mendivelso F. Atención de brotes y epidemias: a propósito del COVID-I9. Revista Médica Sanitas. 2020 p. 4-5. https://doi.org/:I0.26852/0I234250.46

7. Prachand VN, Milner R, Angelos P, Posner MC, Fung JJ, Agrawal N, et al. Medically necessary, time-sensitive procedures: Scoring system to ethically and efficiently manage resource scarcity and provider risk during the COVID-I9 pandemic. J Am Coll Surg. 2020 Apr;9. https://doi.org/IO.IOI6/j.jamcollsurg.2020.04.0II

8. LEIDRAAD. Pre-operative work-up for COVID-ı9 infection in asymptomatic patients scheduled for surgery under general anesthesia. Versie 3.I. [Internet]. 2020 p. I-IO. Fecha de consulta: 2020 May I. Disponible en: https://www.demedischspecialist.nl/sites/default/files/ leidraad_preoperatieve_workup.pdf

9. Al-Muharraqi MA. Testing recommendation for COVID-I9 (SARS-CoV-2) in patients planned for surgery - continuing the service and "suppressing" the pandemic. Br J Oral Maxillofac Surg. 2020 Apr I3. [Epub ahead of print]. https://doi.org/IO.IoI6/j.bjoms.2020.04.0I4

Io. American College of Surgeons, American Society of Anesthesiologists, Association of periOperative Registered Nurses, American Hospital Association. Joint Statement: Roadmap for resuming elective surgery after COVID-I9 pandemic. 2020. p. I-4. Fecha de consulta: 2020 May I. Disponible en: https://www.asahq.org/ about-asa/newsroom/news-releases/2020/04/joint-statement-on-elective-surgery-after-covid-r9-pandemic

II. European Centre for Disease Prevention and Control. Contact tracing: Public health management of persons, including healthcare workers, having had contact with COVID-I9 cases in the European Union - second update, 8 April 2020. Stockholm. ECDC. 2020. p. I-7. Fecha de consulta: 2020 May I. Disponible en: https:// www.ecdc.europa.eu/en/covid-I9-contact-tracing-public-health-management

I2. World Health Organization. W. "Immunity passports" in the context of COVID-I9. 2020. Fecha de consulta: 2020 May I. Disponible en: https:/www.who.int/ news-room/commentaries/detail/immunity-passports-in-the-context-of-covid-I9

I3. Coccolini F, Tartaglia D, Puglisi A, Giordano C, Pistello $\mathrm{M}$, Lodato M, et al. SARS-CoV-2 is present in peritoneal fluid in COVID-I9 patients. [Internet]. Ann Surg. 2020. p. I-5. Fecha de cosulta: 7 de mayo de 2020. Disponible en: https://journals.lww.com/annalsofsurgery/Documents/SARS-CoV-2 is present in peritoneal fluid in COVID-I9 patients.pdf

I4. Brown TW, Chen W, Casanova LM. Survival and disinfection of an enveloped surrogate virus on Tyvek suits used for health care personal protective equipment. Am J Infect Control. 20I6;44:I734-5. https://doi.org/Io.IoI6/j.ajic.20I6.04.247

I5. Cook TM. Personal protective equipment during the coronavirus disease (COVID) 2019 pandemic - a narrative review. Anaesthesia. 2020 Apr;I-I3. https://doi.org/IO.IIII/anae.I507I

I6. Pompeii LA, Kraft CS, Brownsword EA, Lane MA, Benavides E, Rios J, et al. Training and fit testing of health care personnel for reusable elastomeric halfmask respirators compared with disposable N95 respirators. JAMA. 2020 Mar;I-3. https://doi.org/IO.IOoI/ jama.2020.4806

17. American College of Surgeons. Local resumption of elective surgery guidance. American College of Surgeons. 2020. p. I-7. Fecha de consulta: I de mayo de 2020. Disponible en: https://www.facs.org/covid-I9/ clinical-guidance/resuming-elective-surgery

I8. Jinwei H, Feldman L, Abu-Hilal M. Returning to Operations After COVID-I9. SAGES. 2020. Fecha de consulta: I de mayo de 2020. Disponible en: https://www.sages. org/video/returning-to-operations-after-covid-r9/

19. Asociación Española de Cirujanos. Recomendaciones de gestión para la vuelta a la normalidad y "desescalada" en los Servicios de Cirugía general en el contexto de la pandemia COVID-19. 2020. p. I-23. Fecha de consulta: I de mayo de 2020. Disponible en: https:// www.aecirujanos.es/files/noticias/I52/documentos/ Vuelta_a_la_normalidad_v2.pdf

20. American Enterprise Institute. National coronavirus response: A road map to reopening American Enterprise Institute. 2020 Fecha de consulta: I de mayo de 2020. Disponible en: https://www.aei.org/research-products/report/national-coronavirus-response-a-road-map-to-reopening/

2I. The Wall Street Journal. Dr. Anthony Fauci on how life returns to normal - The Journal. WSJ Podcasts. WSJ. Fecha de consulta: 7 de abril de 2020. Disponible en: https://www.wsj.com/podcasts/the-journal/dr-anthony-fauci-on-how-life-returns-to-normal/d57549697027-431e-89fa-eI2788ed9879

22. Institute for Health Metrics and Evaluation. COVID-I9 projections. IHME. 2020 Fecha de consulta: 29 de abril de 2020. Disponible en: https://covidı9.healthdata.org/

23. University of Pennsylvania. COVID-I9 hospital impact model for epidemics (CHIME). 2020 Fecha de consulta: 2 de mayo de 2020. Disponible en: https://penn-chime.phl.io/

24. American College of Surgeons. COVID-r9: Guidance for triage of non-emergent surgical procedures. American College of Surgeons. 2020 p. I-38. Fecha de con- 
sulta: 27 de marzo de 2020. Disponible en: https://www. facs.org/COVID-I9/clinical-guidance/triage

25. Prabhakar A, Helander E, Chopra N, Kaye AJ, Urman RD, Kaye AD. Preoperative assessment for ambulatory surgery. Curr Pain Headache Rep. 20I7;21:I-43. https://doi.org/IO.I007/sII9I6-0I7-0643-7

26. Sterpetti A V. COVID-I9 diffusion capability is its worst, unpredictable chracateristic. How to visit a patient from a distance. Br J Surg. 2020 Apr 24. [Online ahead of print]. https://doi.org/Io.IOo2/bjs.II644

27. Tao KX, Zhang BX, Zhang P, Zhu P, Wang GB, Chen $\mathrm{XP}$. Recommendations for general surgery clinical practice in 2019 coronavirus disease situation. Zhonghua Wai Ke Za Zhi. 2020;58:I70-7. https://doi.org/I0.376o/ cma.j.issn.0529-5815.2020.03

28. Slim K, Theissen A, Raucoules-Aime M. Risk management in ambulatory and short-stay gastrointestinal surgery. J Visc Surg. 2016;153:55-60. https://doi.org/IO.IOI6/j.jviscsurg.2015.I2.002

29. Richards SJG, Frizelle FA, Geddes JA, Eglinton TW, Hampton MB. Frailty in surgical patients. Int J Colorectal Dis. 2018;33:1657-66. https://doi.org/I0.IO07/s00384-0I8-3163-y

30. Wolfel R, Corman VM, Guggemos W, Seilmaier M, Zange S, Muller MA, et al. Virological assessment of hospitalized patients with COVID-20I9. Nature. 2020 Apr I. Online ahead of print. https://doi.org/IO.IO38/s41586-020-2I96-X

3I. Wu Y, Guo C, Tang L, Hong Z, Zhou J, Dong X, et al. Prolonged presence of SARS-CoV-2 viral RNA in faecal samples. Lancet Gastroenterol Hepatol. 2020;5:434-5. Epub 2020 Mar 20. https://doi.org/IO.IoI6/S2468-I253(20)30083-2

32. Baggish MS, Poiesz BJ, Joret D, Williamson P, Refai A. Presence of human immunodeficiency virus DNA in laser smoke. Lasers Surg Med. I991;II:197-203. https://doi.org/IO.IOO2/lsm.I900IIO3O2

33. Weld KJ, Dryer S, Ames CD, Cho K, Hogan C, Lee M, et al. Analysis of surgical smoke produced by various energy-based instruments and effect on laparoscopic visibility. J Endourol. 2007;2I:347-5I. https://doi.org/IO.Io89/end.2006.9994

34. Rajan N, Joshi GP. The COVID-I9: Role of ambulatory surgery facilities in this global pandemic. Anesth Analg. 2020 Apr I;IO.I2I3/ANE.oooooooooooo4847. Online ahead of print. https://doi.org/IO.I213/ANE.ooo 0000000004847

35. Ministerio de Salud y Protección Social de Colombia. Plan de acción para la prestación de servicios de salud durante las etapas de contención y mitigación de la pandemia por SARS-CoV-2 (COVID-I9). 2020. Fecha de consulta: 3I de marzo de 2020. Disponible en: https://www.minsalud.gov.co/Ministerio/Institucional/ Procesos y procedimientos/PSSSoI.pdf

36. Chew NWS, Lee GKH, Tan BYQ, Jing M, Goh Y, Ngiam NJH. A multinational, multicentre study on the psychological outcomes and associated physical symptoms amongst healthcare workers during COVID-I9 outbreak. Brain Behav Immun. 2020 Apr 21;So889I59I(20)30523-7. Online ahead of print. https://doi.org/IO.IOI6/j.bbi.2020.04.049 


\section{Anexo 1. Consentimiento informado recomendado.}

\section{Consentimiento informado sobre riesgo de contagio de COVID-19 (Procedimientos quirúrgicos y/o diagnósticos programados)}

Fecha: $\underline{d d}$ de $m m$ del aaaa en la ciudad de

Nombre del paciente:

Tipo de identificación: $\mathrm{CC}$ $\mathrm{CE}$ Otro $\mathrm{N}^{\circ}$

Yo (nombre completo), identificado con CC $\mathrm{CE}$ de (Ciudad), en pleno uso de mis facultades mentales, actuando en nombre propio (nombre completo del paciente) y con el fin de recibir el tratamiento/procedimiento o en representación de MANIFIESTO:

I. Que en forma LIBRE Y VOLUNTARIA contesto el cuestionario que se plantea a continuación, siendo informado de la necesidad de dar respuesta precisa y veraz a cada uno de los interrogantes, por ser de vital importancia para definir el plan de manejo y las medidas a adoptar para la protección del paciente, el personal de la salud y la comunidad en general:

A. Durante las últimas 2 semanas:

I. ¿Usted, alguien con quien convive o con quien ha sostenido contacto estrecho ha presentado fiebre de $38^{\circ} \mathrm{C}$ o más? SI $\mathrm{NO}$

2. ¿Usted, alguien con quien convive o con quien ha sostenido contacto estrecho ha presentado tos seca? SI__ NO_

3. ¿Usted, alguien con quien convive o con quien ha sostenido contacto estrecho ha presentado dificultad respiratoria? SI $\mathrm{NO}$

4. ¿Usted, alguien con quien convive o con quien ha sostenido contacto estrecho ha estado en contacto con alguien en quien se esté sospechando infección por COVID-I9 o haya sido diagnosticado con esta enfermedad? SI__ NO__

5. ¿Usted, alguien con quien convive o con quien ha sostenido contacto estrecho ha estado fuera del país o en contacto con viajeros provenientes del exterior? SI $\mathrm{NO}$

6. ¿Usted, alguien con quien convive o con quien ha sostenido contacto estrecho ha presentado síntomas tales como diarrea, dolor de garganta, dolores articulares, congestión nasal, reducción o pérdida del olfato o del gusto? SI $\mathrm{NO}$

B. ¿Usted pertenece a algún grupo de riesgo?

I. ¿Es mayor de 65 años? SI__ NO__

2. ¿Vive en un sitio especializado para personas de la tercera edad? SI__ NO__

3. ¿Tiene alteraciones del sistema inmune? SI__ $\mathrm{NO}_{-}$

4. ¿Tiene alguna enfermedad pulmonar crónica (asma, EPOC, etc.)? SI__ NO__ 
5. ¿Tiene hipertensión arterial o enfermedades del corazón? SI__ NO__

6. ¿Tiene obesidad? SI__ NO__

7. ¿Tiene problemas de azúcar (diabetes)? SI__ NO__

8. ¿Tiene alguna enfermedad renal o hepática? SI__ NO_

9. ¿Se encuentra en embarazo? SI__ $\mathrm{NO}_{-}$

II. Que el profesional de la salud me ha explicado que, además de los riesgos propios de la intervención que se realizará, en consideración a la pandemia mundial derivada del SARS-CoV-2 (COVID-I9), actualmente todo procedimiento quirúrgico genera un riesgo de contagio de esta enfermedad, independientemente de que la IPS implemente, como lo ha venido realizando, todos los protocolos de bioseguridad en el quirófano y diferentes áreas hospitalarias y la adecuada utilización de los elementos de protección personal por parte del personal de salud, atendiendo los lineamientos que han dispuesto las autoridades competentes.

III. Que se me ha explicado la naturaleza de la COVID-I9, su sintomatología, modo de contagio, medidas para contenerla y sus complicaciones, frente a lo cual he podido hacer las preguntas relacionadas con la misma, frente a las cuales me han dado respuesta en forma clara y satisfactoria.

IV. Que me comprometo a seguir de manera estricta las instrucciones y recomendaciones que me han sido brindadas para prevenir el riesgo de contagio de la COVID-I9, relacionadas, entre otras, con las medidas de distanciamiento social, lavado de manos y uso de equipos de protección personal que me fueron indicados por el personal de salud.

Dejo constancia, con mi firma, que la información que he brindado corresponde a la realidad, que he comprendido la información que me ha sido suministrada, que he leído y estoy de acuerdo con los términos de este documento y que al suscribirlo estoy dando mi consentimiento y asumiendo los riesgos, deberes y responsabilidades que me han sido explicados.

Firma del paciente o de la persona responsable

$\mathrm{N}^{\circ}$ de documento de

Parentesco*:

"En caso de firma de persona distinta al paciente.

Nombre del profesional de salud

Firma del profesional de salud de

CC: Registro No.:

Cargo: 


\section{Anexo 2. Resúmenes visuales (visual abstract)}

Volver a empezar: Cirugía Electiva durante la pandemia del SARS-CoV2 - COVID-19

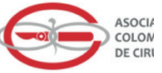

\section{Tamización de los pacientes}

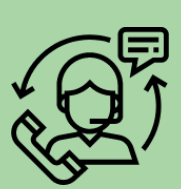

Telemedicina

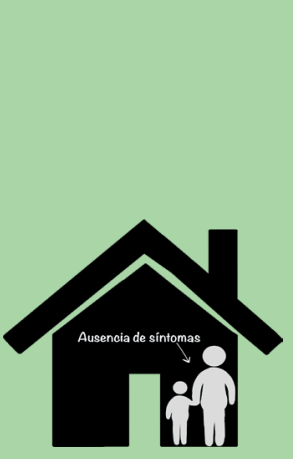

Aislamiento social preoperatorio
Clínico:

- Fiebre

- Odinofagia

- Anosmia

- Disgeusia

- Tos

- Disnea

Adinamia/fatiga

- Síntomas gastrointestinales

- Antecedente de confinamiento

- Contacto 14 días: paciente + o sospechoso

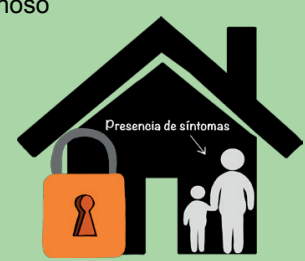

Cancelado

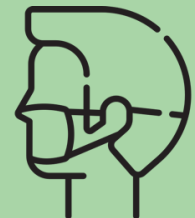

Pruebas:

Moleculares

- PCR

Serológicas

$-\lg G$

$-\lg M$

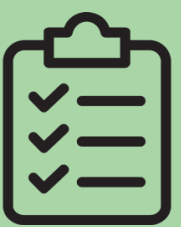

Realizar de acuerdo a disponibilidad institucional

Sensibilidad:

- 32\% 1er día de inicio de síntomas

- 50-70\% día 7-10 VPP $74.5 \%$

VPN $58.1 \%$

En pacientes asintomáticos no hay estudios de sensibilidad de las pruebas

Volver a empezar: Cirugía Electiva durante la pandemia del SARS-CoV2 - COVID-19

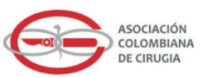

\section{Instalaciones}

1. Disponibilidad:

Camas de UCI

Camas de hospitalización

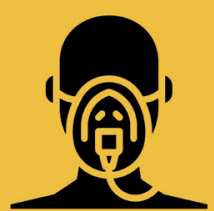

4. Zonas públicas hospitalarias con distanciamiento social de 1-2 metros

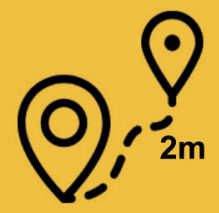

2. Ruta no COVID-19: cirugía electiva

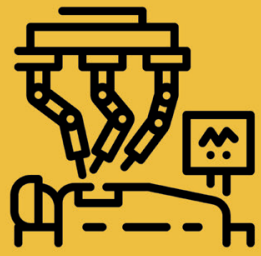

5. Capacidad de expandir las áreas de $\mathrm{UCl}$ y hospitalización

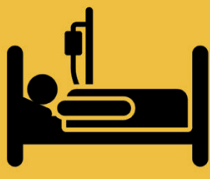

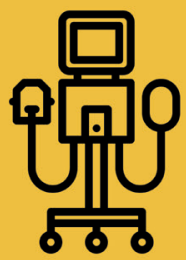

3. Ruta COVID-19: cirugía de urgencias

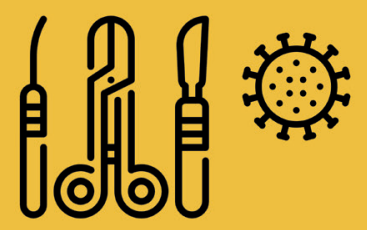

6. Cancelación del programa de cirugía electiva según la evolución de la pandemia

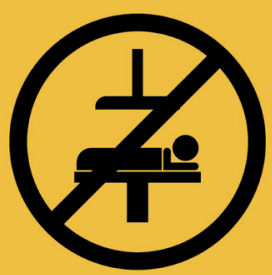


Volver a empezar: Cirugía Electiva durante la pandemia del SARS-CoV2 - COVID-19
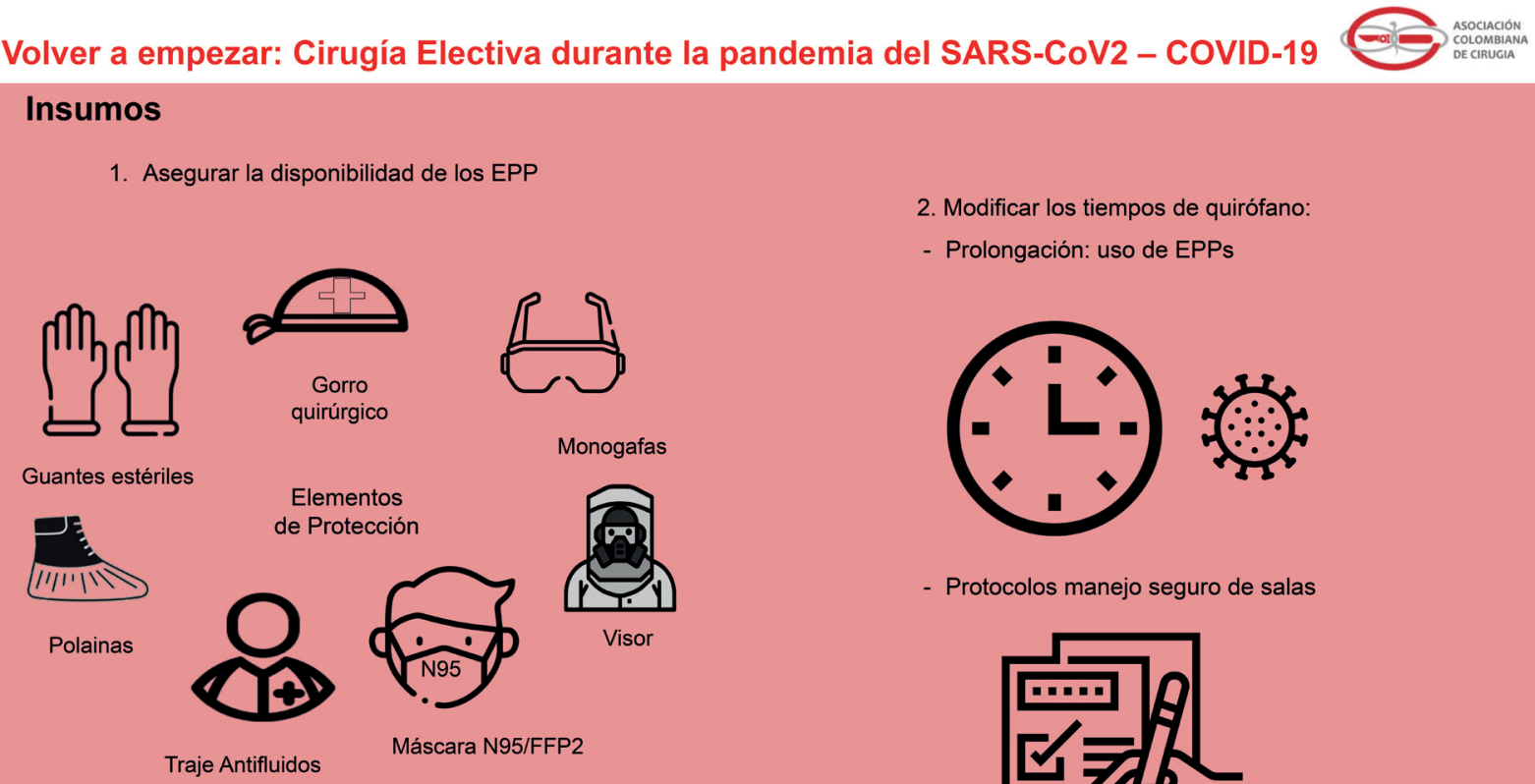

2. Modificar los tiempos de quirófano:

- Prolongación: uso de EPPs

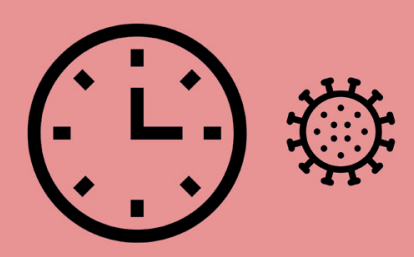

- Protocolos manejo seguro de salas

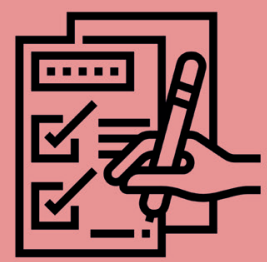

Volver a empezar: Cirugía Electiva durante la pandemia del SARS-CoV2 - COVID-19

\section{ASOCIACÍ́N
COLOMBOANA
DE CRUCIA}

\section{Perioperatorio}

1. Consentimiento informado específico con riesgo COVID-19

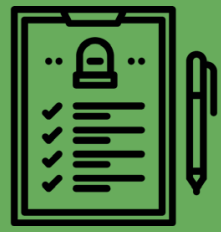

4. Revistas y juntas virtuales

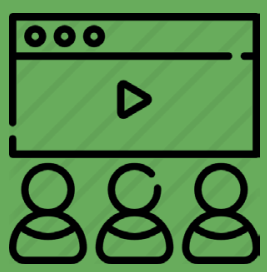

2. Telemedicina: valoraciones pre y postquirúrgica

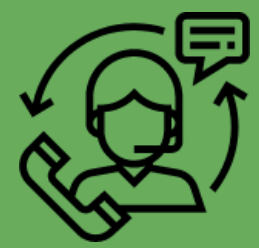

5. Mantener el bienestar del personal de salud

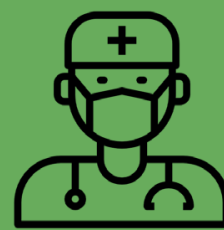

3. Restringir elementos en salas: celulares, parlantes y cámaras

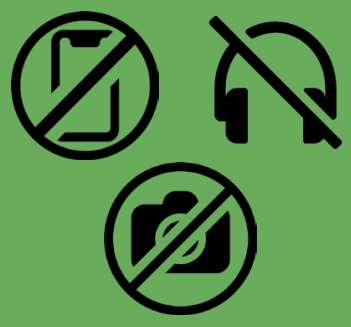

6. Actualización permanente

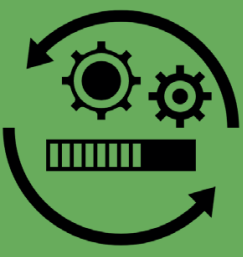


Volver a empezar: Cirugía Electiva durante la pandemia del SARS-CoV2 - COVID-19

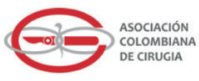

Riesgos de contacto en el proceso de cirugía ambulatoria

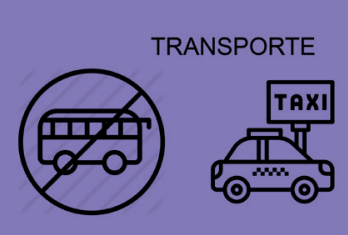

Evitar transporte público masivo

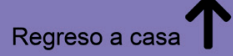

RECUPERACIÓN

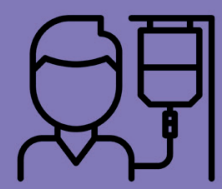

Elementos de protección Distancia aumentada Menos pacientes en sala

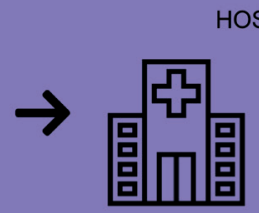

Minimizar contactos

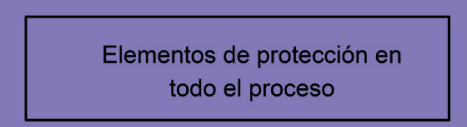

PROCEDIMIENTO QUIRÚRGICO

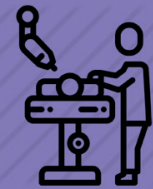

Equipos de protección, ruta segura Mínimo personal en la sala Minimo material en sala
ADMISIONES Y REGISTRO

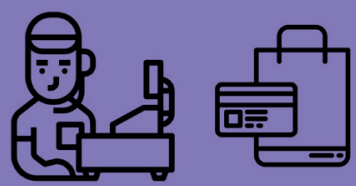

Minimo personal, automatizado/virtual proceso ágil y corto

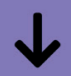

INGRESO A SALAS
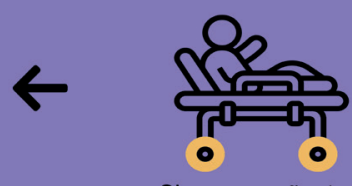

Sin acompañante

Ruta segura 